\title{
DETERMINAÇÃO DE FUNÇÃO COVARIÂNCIA LOCAL PARA A PREDIÇÃO DE ANOMALIAS DA GRAVIDADE BOUGUER E VALORES DA GRAVIDADE VISANDO À OBTENÇÃO DE NÚMEROS GEOPOTENCIAIS
}

Determination of local covariance function for the prediction of the Bouguer gravity anomalies in order to obtain geopotential numbers

\author{
ROGERS ADEMIR DRUNN PEREIRA ${ }^{1}$ \\ HENRY MONTECINO CASTRO ${ }^{2}$ \\ SÍLVIO ROGÉRIO CORREIA DE FREITAS ${ }^{2}$ \\ QUINTINO DALMOLIN ${ }^{2}$ \\ VAGNER GONÇALVES FERREIRA ${ }^{3}$ \\ ${ }^{1}$ Universidade Federal do Pampa - UNIPAMPA \\ Rua Luiz Joaquim de Sá Brito, s/n - Bairro Promorar \\ Itaqui/RS - CEP 97650000 \\ r51505150@gmail.com \\ ${ }^{2}$ Programa de Pós-Graduação em Ciências Geodésicas \\ Universidade Federal do Paraná - Curitiba - Brasil \\ henrymontecino@gmail.com; sfreitas@ufpr.br; qdalmolin@ufpr.br \\ ${ }^{3}$ School of Earth Sciences and Engineering \\ Hohai University - Nanjing - China \\ vagnergf@hhu.edu.cn
}

\begin{abstract}
RESUMO
Considerando as dimensões de um país como o Brasil, realizar observações gravimétricas sobre todas as linhas de nivelamento do país ainda constitui-se um problema pertinente quando o objetivo é a determinação de números geopotenciais e/ou quantidades relacionadas ao campo da gravidade (e.g. anomalias da gravidade). Funções de covariância locais foram construídas a partir de valores da gravidade disponibilizados pelo Instituto Brasileiro de Geografia e Estatística (IBGE). Estes valores foram digitalizados e anexados à base de dados do Laboratório de Referenciais Geodésicos e Altimetria por Satélites (LARAS) da UFPR, a qual já contém dados da rede gravimétrica argentina. Estas funções de covariância foram desenvolvidas para anomalias da gravidade Bouguer na região
\end{abstract}


fronteiriça Brasil/Argentina. Estudos com funções polinomiais e de Fourier foram avaliadas utilizando dez por cento dos pontos originais para checagem. Os resultados obtidos em termos de Erro Médio Quadrático (RMS) para a função polinomial de quarta ordem e para a função de Fourier de terceira ordem foram do nível do mGal.

Palavras-chave: Função Covariância; Colocação por Mínimos Quadrados; Números Geopotenciais.

\section{ABSTRACT}

Because of the impossibility of gravimetric observations on all over the leveled lines, the knowledge of the gravity quantities is still a problem when the subject is, for example, the computation of geopotential numbers. Local Covariance Functions (LCF) were generated from gravity values of Brazilian Institute of Geography and Statistics that were digitized and added to Geodetic Reference Systems and Satellite Altimetry Laboratory (LARAS) geodetic database. This base contains gravimetric and altimetric points provided by IGN (Argentina) too. The LCFs were developed for gravity Bouguer anomalies to points which heights were available and the gravity values were recovered. These points are located on the Brazil-Argentina's border. Studies with polynomial function and Fourier function were tested with ten per cent of points of database reserved to testing and the polynomial fourth degree function showed the best results (about $1 \mathrm{mGal}$ ).

Keywords: Covariance Functions; Least Squares Collocation; Geopotential Numbers.

\section{INTRODUÇÃO}

A rede vertical brasileira foi materializada a partir de observações provenientes de nivelamento geométrico corrigidas do campo da gravidade normal, resultando no que hoje é conhecida como uma rede de altitudes ortométricas-normal (HECK ${ }^{1}, 2003$ apud FERREIRA, 2011). Esse sistema de altitudes não é adequado quando se deseja obter altitudes com significado físico. Com vistas à modernização do sistema de altitudes, é imprescindível a utilização de valores da gravidade associados aos desníveis provenientes do nivelamento geométrico, os quais possibilitam o cálculo de diferentes tipos de altitudes e contribuem para o cálculo do geóide local.

Infelizmente, além da baixa resolução gravimétrica no Brasil, muitas das observações gravimétricas não foram realizadas sobre pontos pertencentes às linhas de nivelamento. Assim, o processo de predição de valores da gravidade no Brasil ainda é um problema a ser solucionado, seja para a geração de números

${ }^{1}$ HECK, B. Rechenverfahren un Auswertemodelle der Landesvermessung. 3rd. ed.Karlsruhe: Wichman, 2003.

Bol. Ciênc. Geod., sec. Artigos, Curitiba, v. 17, nº 2, p.238-253, abr-jun, 2011. 
geopotenciais, seja para o cálculo do geóide, seja para a conexão de diferentes data verticais. Em especifico, como no caso da conexão do Marégrafo de Mar Del Plata, origem do sistema vertical argentino com o Marégrafo de Imbituba, origem do sistema vertical brasileiro. No contexto da densificação das observações da gravidade, salienta-se que a existência de uma boa densidade com distribuição regular contribui para a solução de data verticais nacionais com abordagem baseada no Problema de Valor de Contorno da Geodésia (PVCG) fixado (ZHANG et al., 2008). A situação das observações da gravidade utilizadas neste trabalho está apresentada na Figura 1, onde claramente nota-se a falta de homogeneidade espacial, a qual pode ser conseguida mediante o processo de Colocação por Mínimos Quadrados (CMQ).

Figura 1 - Valores da gravidade conhecidos na região de estudos, à esquerda; pontos de controle utilizados no cálculo do RMS, à direita.

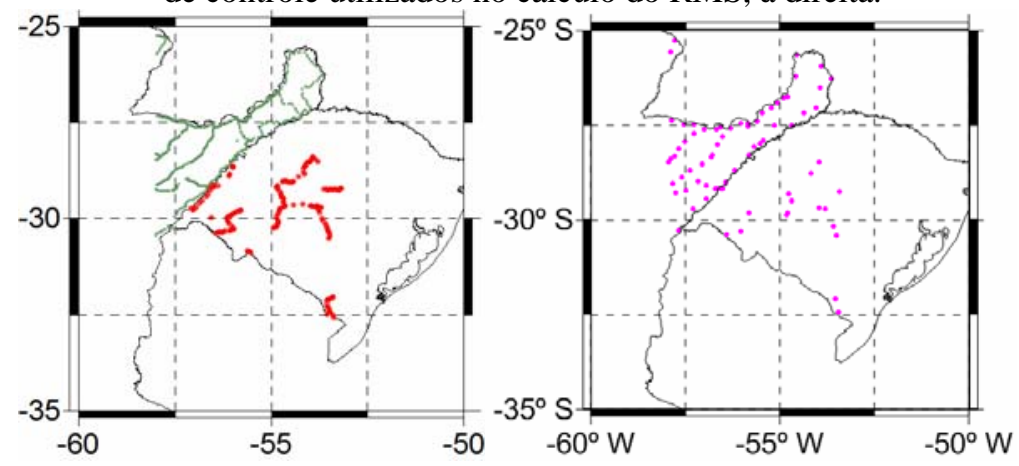

A predição de valores de anomalias da gravidade tem sido alvo de pesquisa nas áreas da Geodésia e da Geofísica através de diversas técnicas como as Redes Neurais Artificiais (TIERRA \& DE FREITAS, 2005) como a Colocação por Mínimos Quadrados e ainda em seus casos mais específicos, como a Krigagem (VERMEER, 2010, p. 98). Neste artigo, apresentar-se-á a predição de anomalias da gravidade de Bouguer utilizando Colocação por Mínimos Quadrados seguida do processo de recuperação da gravidade. Para tal serão necessárias coordenadas geodésicas e altitudes dos pontos de onde se deseja efetuar a predição assim como uma base de dados que contenha a gravidade, a altitude e as coordenadas geodésicas de pontos representativos da região.

\section{METODOLOGIA}

\subsection{Região de Estudos}

Tomando-se como referência a fronteira do Brasil com a Argentina no estado do Rio Grande do Sul e a base de dados disponível junto ao LARAS, a região de 
estudos é definida entre os limites de Latitude $35^{\circ} \mathrm{S}$ e $25^{\circ} \mathrm{S}$ e Longitude entre $60^{\circ} \mathrm{W}$ e $50^{\circ} \mathrm{W}$.

Os valores da gravidade do lado argentino foram disponibilizados pelo Instituto Geográfico Nacional da Argentina, enquanto que os valores da gravidade na fronteira oeste do Rio Grande do Sul são oriundos de Campanhas realizadas pelo LARAS; os demais dados provieram do Instituto Brasileiro de Geografia e Estatística (IBGE) disponível na internet. Estes dados estão representados na Figura 1, onde também encontram-se os pontos de controle para o cálculo do RMS, É importante esclarecer que o IBGE disponibiliza um número maior de estações gravimétricas. No entanto, utilizaram-se somente as estações que até a data de aquisição (Setembro/2010) possuíam altitude.

\subsection{Características dos Dados Disponíveis}

Do Instituto Geografico Nacional (IGN) - Argentina - foram utilizados 559 pontos com valores de gravidade e altitude referida ao Datum Vertical Argentino de Mar Del Plata. Destes, reservou-se 56 para checar a validade das funções. Do lado brasileiro foram digitalizados 1.222 pontos gravimétricos extraídos do Banco de Dados Geodésicos do Instituto Brasileiro de Geografia e Estatística (IBGE), uma vez que estes encontravam-se em formato não editável. Dentre estes, apenas 157 continham o valor de altitude referida ao Datum Vertical de Imbituba. Ainda, adicionou-se 25 pontos de gravidade observados pela UFPR, reservando 18 pontos do lado brasileiro para a checagem e validação das funções. No total, portanto, foram reservados 74 pontos para checagem do RMS. Em virtude da forte correlação com o terreno e a pouca suavidade das anomalias free-air, calculou-se as anomalias de Bouguer, as quais apresentaram características suaves e pouco dependentes do terreno.

Neste artigo, a gravidade normal foi considerada como sendo a parte sistemática do modelo e as anomalias de Bouguer como sendo o sinal. A heterogeneidade das fontes de informações gravimétricas impossibilitou a estimação das covariâncias do ruído. Assim, todas as observações utilizadas foram consideradas como isentas de erro. Esta condição dispensa a subtração da parte sistemática, apresentada na (05), na próxima seção e também facilita a obtenção da covariância empírica, uma vez que não se conhece os desvios padrões das observações da gravidade realizadas pelo IBGE. Somente com os desvios-padrão das observações é possível calcular a matriz variância-covariância das observações. Assim, considerar as observações como isentas de erro dispensa o cálculo de uma matriz adicional que não gera diferença no ajuste das funções utilizadas, lembrando que os pesos não interferem na determinação dos coeficientes daquelas funções.

O problema de referencial vertical no cálculo das anomalias exigiu uma compatibilização das altitudes do conjunto de pontos utilizados no cálculo da covariância empírica. A partir de uma determinação executada com nivelamento geométrico de primeira ordem na fronteira do Brasil com a Argentina (cidade de 
Passo de Los Libres), na estação PFA 1XA, obteve-se um afastamento entre as superfícies de nível dos dois sistemas de 0,7193 m entre os referenciais, a qual foi adicionada às altitudes argentinas, gerando um sistema comum referido ao Datum Vertical de Imbituba, cujas coordenadas horizontais estão referidas ao SIRGAS.

\subsection{OBTENÇÃO DAS ANOMALIAS DE BOUGUER E RECUPERAÇÃO DOS VALORES DA GRAVIDADE}

As anomalias de Bouguer são calculadas a partir da equação (01) (HOFMANN-WELLENHOF \& MORITZ, 2005, p.136):

$$
\Delta g_{B}=g_{B}-\gamma=g+0.1967 H-\gamma
$$

onde $\Delta g_{B}$ é a anomalia Bouguer; $g_{B}$ é a gravidade reduzida do platô de Bouguer, sobre o geóide; $\gamma$ é a gravidade normal, sobre o elipsóide de referência, e $H$ é a altitude ortométrica. $g$ é a gravidade observada na superfície física da Terra.

Como se pode notar, as anomalias de Bouguer necessitam da altitude ortométrica $H$ para serem calculadas. Neste trabalho as altitudes fornecidas pelas instituições de referência (IBGE e IGN) foram consideradas em substituição às altitudes ortométricas. Este cálculo considera alguns pressupostos (e.g. densidade da crosta e gradiente da gravidade normal constantes; gradiente da gravidade normal representativo do gradiente da gravidade real) e que podem não refletir a condição real do local. No entanto, o resultado será tratado como aproximação suficiente. Mais discussões a respeito podem ser encontradas, por exemplo, em Kiamehr (2006).

Uma vez obtidas as anomalias de Bouguer, o valor da gravidade pode ser recuperado nos pontos onde é conhecida a altitude isolando-se $g$ na (01). O cálculo da gravidade normal foi efetuado e está implícito na obtenção da anomalia. Aqui, utilizou-se se a Fórmula de Somigliana (BOMFORD, 1980, p. 419). No presente caso as reduções não levaram em conta correções do terreno. O efeito desta quantidade na região estudada pode ser encontrado em Pereira e de Freitas (2010) e atinge um máximo de $0,2 \mathrm{mGal}$. Como as correções do terreno são baixas, dispensou-se o cálculo de anomalias de Bouguer completas, qual seja, considerando-se as correções de terreno.

\subsection{Colocação por Mínimos Quadrados}

A Colocação por Mínimos Quadrados foi desenvolvida originalmente por Helmut Moritz e Torben Krarup visando ajustamento, filtragem e predição de grandezas do campo da gravidade (KRARUP, 2006; MORITZ, 1972). A idéia básica resume-se em, a partir de um funcional conhecido que represente a parte sistemática de uma quantidade física, obter uma função por mínimos quadrados que 
represente o sinal que se deseja predizer e/ou filtrar. As etapas da Colocação por Mínimos Quadrados são dadas por (MORITZ, 1972, p. 7 e Ibid. p. 15):

$$
\begin{gathered}
\mathbf{x}=\mathbf{A X}+\mathbf{s}+\mathbf{n} \\
\mathbf{X}=\left(\mathbf{A}^{T} \mathbf{C}^{-1} \mathbf{A}\right)^{-1} \mathbf{A}^{T} \mathbf{C}^{-1} \mathbf{x}
\end{gathered}
$$

Assim, três aspectos são intrínsecos à Colocação por Mínimos Quadrados. O primeiro deles é o cálculo de uma função covariância empírica (SCHMIDT, 2007, p.34). No segundo, ajusta-se uma função qualquer que modele o comportamento da Covariância Empírica, podendo-se utilizar o ajustamento paramétrico como apresentado na (03). E por último utiliza-se a função ajustada para o cálculo da Matriz Covariância C, função da distância $d$ entre os pontos e apresentada em (04). A etapa de predição é dada pela Equação (05), a seguir:

$$
\begin{gathered}
\mathbf{C}=\left\lfloor C_{i j}\right\rfloor ; C_{i j}=f(d) \\
\mathbf{s}=\mathbf{C}_{S X} \mathbf{C}^{-1}(\mathbf{x}-\mathbf{A X})
\end{gathered}
$$

Nas equações acima, $\mathbf{A X}$ representa a parte sistemática; $\mathbf{s}$ o sinal e $\mathbf{n}$ o ruído; $\mathbf{C}$ é a matriz covariância dos pontos conhecidos e $\mathbf{C}_{S X}$ a matriz covariância do ponto a predizer e dos pontos conhecidos e finalmente $\mathbf{x}$ é o vetor das observações.

\subsection{Números Geopotenciais}

O número geopotencial de um ponto $P$ é calculado segundo Torge (1991, p. 42) pela equação:

$$
C_{P}=W_{o}-W_{P}=-\int_{P_{0}}^{P} d W=-\int_{P_{0}}^{P} g d n
$$

onde $W_{0}$ é o geopotencial da superfície de referência, $W_{P}$ o geopotencial do ponto na superfície e $d W$ é a diferença de potencial entre os pontos em questão obtida do produto da gravidade média $g$ entre os dois pontos e o desnível $d n$ entre ambos. Observa-se a impossibilidade da obtenção de números geopotenciais se não for conhecida a gravidade sobre os pontos considerados. Ainda, mesmo que se conheçam as informações da gravidade sobre todos os pontos da rede de nivelamento, apenas diferenças relativas de potencial podem ser obtidas. Esta dificuldade ocorre porque o Datum Vertical ao qual estão vinculadas as observações de nivelamento não materializa $W_{0}$ Um sistema de altitudes baseado em números geopotenciais asseguraria um valor único para cada ponto independentemente do trajeto percorrido, o que atualmente não acontece na rede vertical brasileira.

Dentro do objetivo deste trabalho, coaduna como possível o uso da ferramenta de interpolação para a geração de um termo corretivo aos números esferopotenciais 
$\left(C^{\prime}\right)$ (números geopotenciais normal) a fim de convertê-los em números geopotenciais $C$ como:

$$
C_{P}=C_{P}{ }_{P}+\int_{P_{0}}^{P} \delta g d n
$$

em que $\delta g$ é o distúrbio de gravidade calculado pela diferença entre a gravidade observada e a gravidade normal para o mesmo ponto em consideração.

\subsection{Funções Utilizadas para o Ajuste da Covariância Empírica}

Apesar de explorar outros tipos de funcionais (como por exemplo as exponenciais, racionais, gaussianas), as funções que serão tratadas neste artigo são a) a Função de Hirvonen, pela sua importância histórica e b) as Funções polinomial de $4 .^{\text {a }}$ ordem e de Fourier de $3 .^{a}$ ordem, por fornecerem os melhores ajustes. Estas funções estão expressas a seguir. Em todos os casos $s$ é a variável independente, cujo significado físico é a distância de correlação entre as variáveis.

A função de Hirvonen é dada pela (08), a seguir.

$$
\operatorname{COV}_{1}=\frac{C_{0}}{\left(1+\left(\frac{s}{d}\right)^{2}\right)}
$$

A função de Fourier de 3. ${ }^{a}$ ordem é dada por

$$
\begin{aligned}
\operatorname{COV}_{2}=a_{0}+ & a_{1} \cdot \cos (s \cdot w)+b_{1} \cdot \operatorname{sen}(s \cdot w) \\
+ & a_{2} \cdot \cos (2 \cdot s \cdot w)+b_{2} \cdot \operatorname{sen}(2 \cdot s \cdot w) \\
& +a_{3} \cdot \cos (3 \cdot s \cdot w)+b_{3} \cdot \operatorname{sen}(3 \cdot s \cdot w)
\end{aligned}
$$

A função polinomial de $4 .^{\circ}$ grau é dada pela (10).

$$
\operatorname{COV}_{3}=p_{1} \cdot s^{4}+p_{2} \cdot s^{3}+p_{3} \cdot s^{2}+p_{4} \cdot s+p_{5}
$$

\section{RESULTADOS E DISCUSSÃO}

\subsection{Covariância Empírica e Funções Ajustadas}

Após o ajuste de funções do tipo exponenciais, gaussianas, racionais, de Fourier e Polinomiais, variando-se as suas ordens, chegou-se às funções de Fourier de $3 .^{\text {a }}$ ordem e polinomial de $4 .^{\text {a }}$ ordem como melhor ajustadas à covariância empírica. 
A covariância empírica (empirical covariance), representada nas Figuras 2, 3, 4 e 5 e 7 foi calculada a partir das anomalias da gravidade Bouguer “observadas":

Figura 2 - Covariância Empírica.

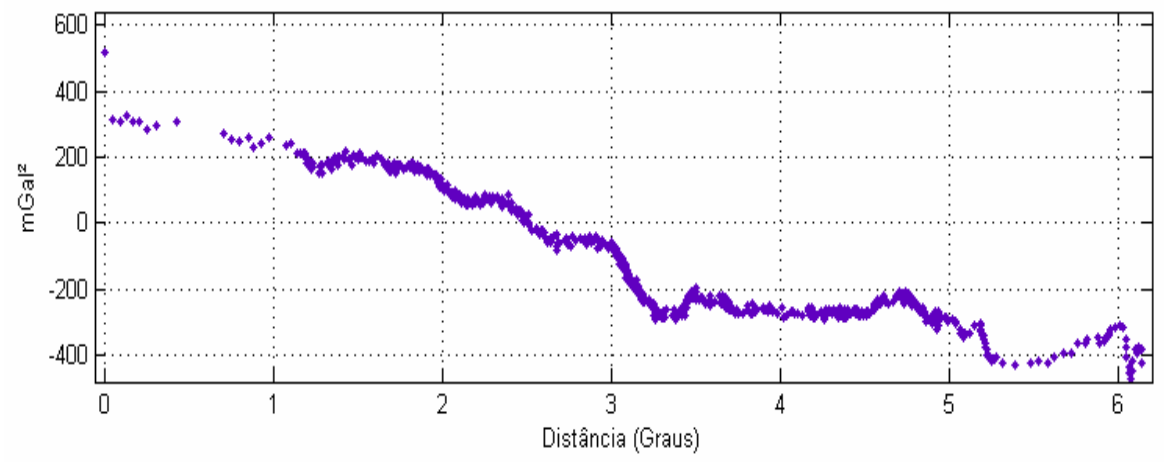

A covariância empírica foi calculada segundo Heiskanen \& Moritz (1967, Seção 7.2). Para ajustar a covariância empírica, inicialmente utilizou-se uma equação do tipo da (08), atribuída geralmente a Hirvonen (KOTSAKIS, 2007). O termo independente daquela função é a distância $d$.

O ajuste dos dados à Função de Hirvonen (Hirvonen Function) pode ser visualizado na Figura 3, abaixo.

Figura 3 - Ajuste da Função de Hirvonen com os dados da covariância empírica.

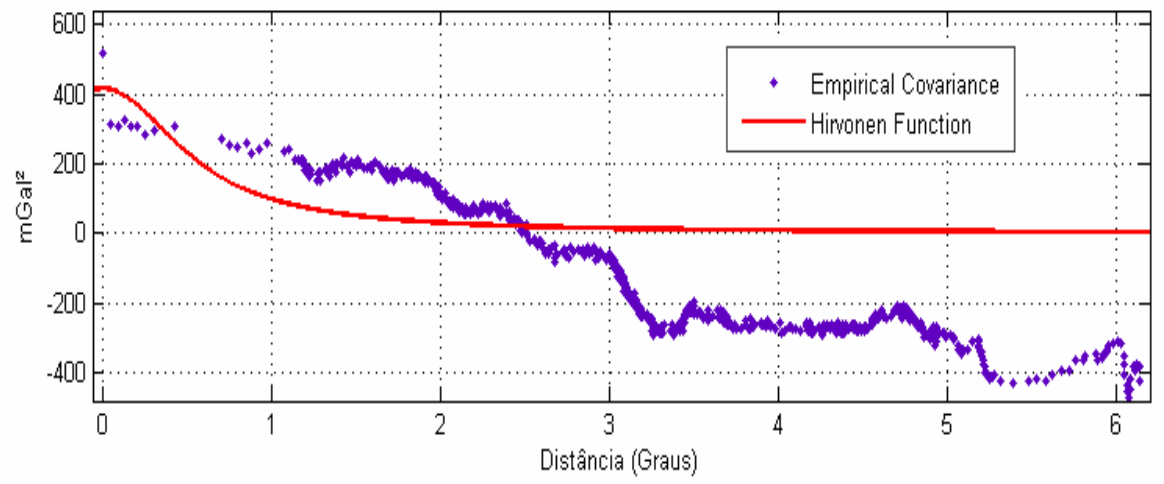

A covariância empírica foi ajustada também através da função de Fourier de Terceira ordem (F3) descrita pela (09) e cujo resultado está apresentado Figura 4. O Termo independente neste caso é a variável $s$. Este termo $s$ não deve ser confundido com o sinal s. 
Figura 4 - Ajuste de uma função de Fourier de 3. ${ }^{a}$ ordem com os dados da covariância empírica.

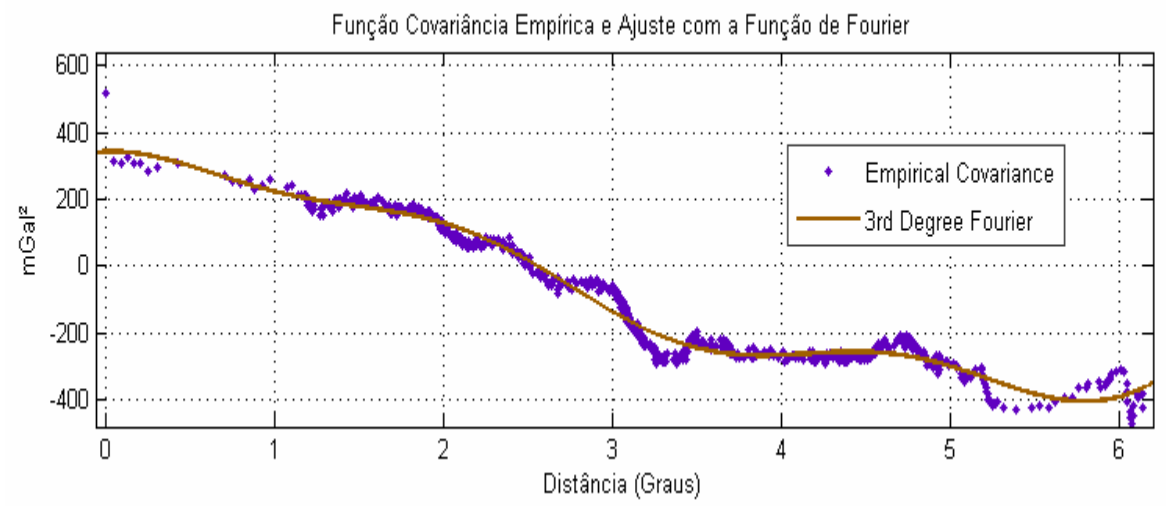

E, por último, utilizou-se uma função polinomial de 4 . $^{\text {a }}$ ordem (P4), descrita em (10) para o ajuste da covariância empírica e o resultado é apresentado na Figura 5. Também para esta última função, o termo independente é a variável $s$.

Figura 5 - Ajuste de uma função polinomial de $4 .^{\text {a }}$ ordem com os dados da covariância empírica.

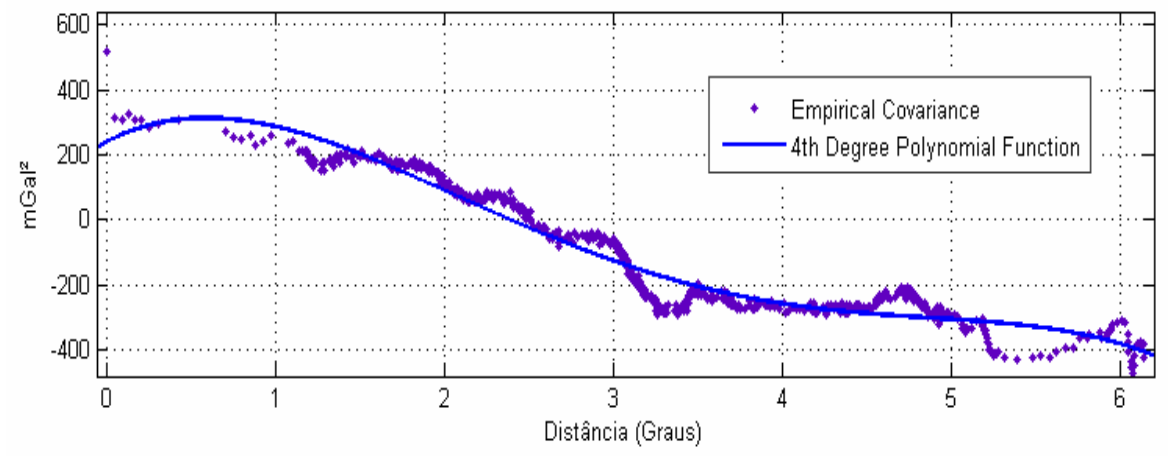

Os coeficientes obtidos no ajustamento para as três funções e do teste que estima a qualidade das mesmas $\left(\mathrm{R}^{2}\right)$ estão apresentados na Tabela 1 . Na figura 6, além dos resíduos das três funções ajustadas, apresentam-se também os resíduos da função "Sanchez", a qual não foi abordada no texto, mas será discutida na seção 3.1.1. 
Tabela 1 - Ajustes das Funções de Hirvonen, P4 e F3 para a função covariância empírica.

\begin{tabular}{|c|c|c|c|c|c|}
\hline \multicolumn{2}{|c|}{ Hirvonen } & \multicolumn{2}{|c|}{$\begin{array}{c}\text { Função Polinomial de } 4 .^{\text {a }} \\
\text { Ordem (P4) }\end{array}$} & \multicolumn{2}{|c|}{$\begin{array}{c}\text { Função de Fourier de } 3{ }^{\circ} \\
\text { Ordem (F3) }\end{array}$} \\
\hline $\begin{array}{r}\mathrm{C}_{0} \\
\mathrm{~d}\end{array}$ & $\begin{array}{l}415.3 \\
0.5548\end{array}$ & $\begin{array}{l}\mathrm{p}_{1} \\
\mathrm{p}_{2} \\
\mathrm{p}_{3} \\
\mathrm{p}_{4} \\
\mathrm{p}_{5}\end{array}$ & $\begin{array}{l}-4.56 \\
66.42 \\
-278.5 \\
264.6 \\
240.3\end{array}$ & $\begin{array}{l}a_{0} \\
a_{1} \\
b_{1} \\
a_{2} \\
b_{2} \\
a_{3} \\
b_{3} \\
\text { w }\end{array}$ & $\begin{array}{l}-48.56 \\
268.4 \\
198.4 \\
85.14 \\
12.27 \\
35.7 \\
-64.65 \\
0.7906\end{array}$ \\
\hline \multicolumn{2}{|c|}{$\mathrm{R}^{2}=-0.2285$} & \multicolumn{2}{|c|}{$R^{2}=-0.9508$} & \multicolumn{2}{|c|}{$\mathrm{R}^{2}=-0.9765$} \\
\hline
\end{tabular}

Figura 6 - Resíduos das funções ajustadas.

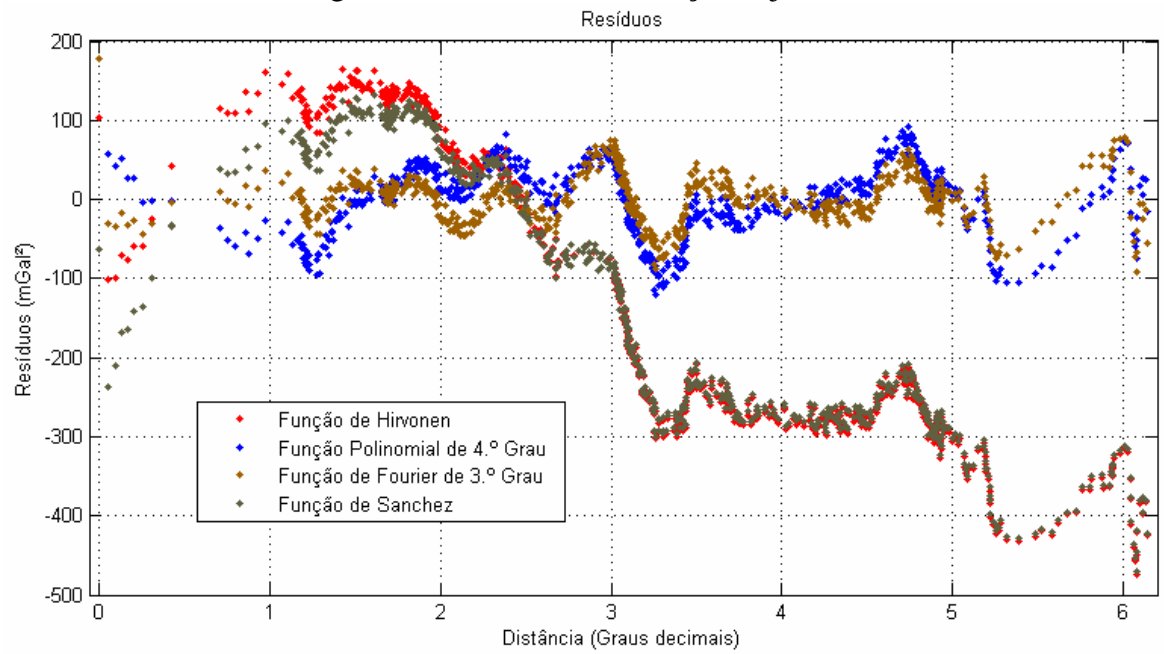

\subsubsection{Considerações sobre o PREDGRAV}

O PREDGRAV é um programa que se vale da Colocação por Mínimos Quadrados e serve à predição de valores associados com a gravidade, adotado pelo Sistema de Referência Geocêntrico para as Américas (SIRGAS). Este programa é a solução indicada para a predição de anomalias da gravidade na América do Sul. Em comunicação pessoal com Laura Sánchez do Deutsches Geodaetisches Forschungsinstitut (DGFI), representante do grupo que trabalha naquele sistema de 
referência, esta apresentou de próprio punho a função utilizada por aquele programa, denominada Função de Sánchez, descrita a seguir:

$$
\mathrm{COV}_{4}=a \cdot e^{-b \cdot S}
$$

Nos resultados da seqüência, $a$ e $b$ foram considerados os parâmetros a ajustar e $S$ a variável independente. Então, ajustou-se a Função de Sánchez para os dados conhecidos e plotou-se este ajuste na mesma figura que contém a covariância empírica, na Figura 7, a seguir.

Figura 7 - Função Sánchez ajustada à Covariância Empírica.

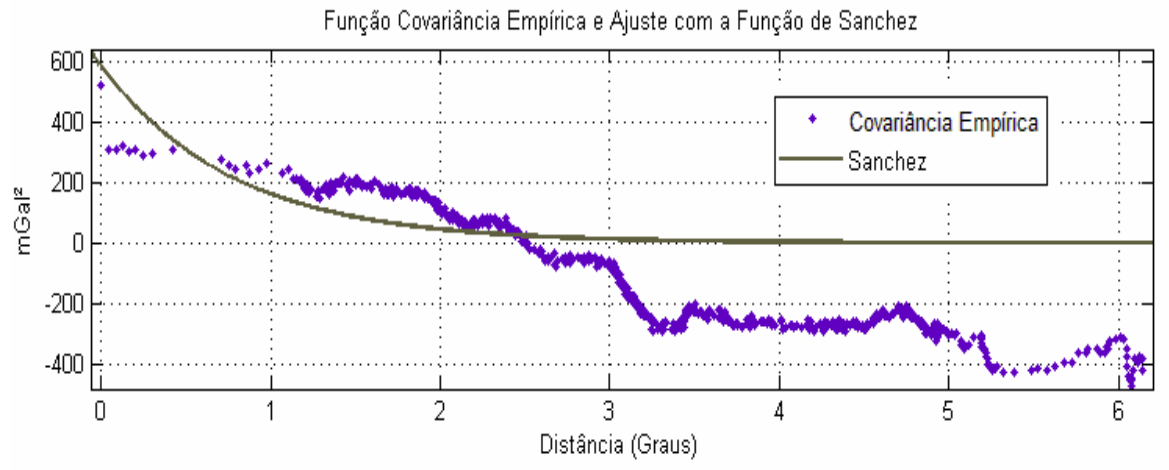

O resíduo para este ajuste foi apresentado na Figura 6. O R $\mathrm{R}^{2}$ estimado foi de 0,1668, de onde se pode concluir o mau ajuste da função. A partir deste estimador, pode-se inferir que existe má-aderência da função Sánches à Covariância Empírica apresentada, desde que realizadas as mesmas considerações.

\subsection{Discussão das Funções Covariância Utilizadas e Resultados de Predição}

Analisando os coeficientes das funções apresentados na Tabela 1, percebe-se que as mesmas não podem ser tratadas rigorosamente como positiva-definidas. Os resultados são confirmados ao se fazer uma decomposição espectral ou calcular o determinante da Matriz Covariância C utilizando a (09) ou a (10). No entanto, cabe destacar que este tema não faz parte do escopo inicial deste trabalho. Informações relevantes associadas com o sistema de equações normais da CMQ podem ser encontradas em Moritz (1976) e considerações sobre o uso de funções que não são positiva-definidas em Sünkel (1978). Dificuldades de inversão de matrizes neste trabalho foram superadas utilizando-se a Inversa Generalizada de Moore-Penrose em substituição à Inversa de Cailey. 
Destaque-se que outras funções também poderiam ser ajustadas à Covariância Empírica (CE). As F3 e P4 foram escolhidas com base no comportamento visual diante da CE. No que se refere ao detalhe, o desenvolvimento em baixo grau dos coeficientes das funções foi preferido em substituição ao alto grau visando manter a capacidade de generalização dos coeficientes obtidos.

Diante do $\mathrm{R}^{2}$ obtido, a F3 é melhor representativa da CE relativamente aos demais funcionais testados. Entretanto, baseado nas conclusões, onde são explicitados os valores de comparação entre os dados preditos e os de referência (menor RMS para P4), foi possível perceber que o $\mathrm{R}^{2}$ talvez não seja o melhor indicador de qualidade para a situação apresentada embora tenha sido utilizado como critério para selecionar as F3 e P4 dentro de um conjunto de outras funções. Tal fato pode ter sido causado pela perda de generalização de F3, hipótese apresentada no parágrafo anterior, ou ainda pelo fato de $\mathrm{R}^{2}$ ser um estimador estatístico frente a uma situação cuja homogeneidade espacial e temporal das observações da gravidade envolvidas não é elemento favorável, embora tenha sido tratada como tal.

Recuperando-se o valor da gravidade com a (01), chega-se a um RMS calculado de $1,02 \mathrm{mGal}$ depois de retirados 11 pontos com o filtro $2 \sigma(2.79 \mathrm{mGal})$ para $\mathrm{P} 4$ e comparando-se com os valores observados. A mesma comparação não foi feita com F3. É importante destacar que Luz (2008) apresenta para a mesma região desvios-padrão que variam entre 0 e $20 \mathrm{mGal}$. Para todo o Brasil, o mesmo autor aponta desvios de até $60 \mathrm{mGal}$, o que torna os resultados de predição apresentados aqui significativamente melhores quando comparados com os obtidos via ferramenta recomendada pelo SIRGAS.

Destaque-se que este resultado poderia ser diferente para outras bases de dados. O grande número de combinações de funções (e.g. um polinômio de funções senoidais, ou Funções de Fourier com bases exponenciais) impossibilita dizer se outro funcional poderia resultar em melhores RMS. Por causa disso e da distribuição heterogênea (espacial e temporal) a interpolação de valores da gravidade ainda é um problema que deve ser considerado na América do Sul e que só pode ser avaliado empiricamente. No entanto, os funcionais apresentados alternativamente adquirem importância na medida em que afirmam um RMS relativamente às funções clássicas utilizadas.

A krigagem não foi utilizada como elemento de comparação porque sofre dos mesmos problemas que a CMQ (na verdade há muitas semelhanças entre estes métodos). Se a CMQ necessita de uma função covariância empírica a ser ajustada, na krigagem, o variograma, também ajustado, é um problema a ser superado. Se o variograma não é ajustado, então a solução por krigagem é totalmente equivalente a fixar uma função para a CMQ e tiliza-la indiscriminadamente. As funções utilizadas para a predição segundo a CMQ foram o escopo deste trabalho. Sugerese, portanto, um estudo equivalente ajustando diferentes variogramas para os mesmos pontos apresentados aqui. 
Os resultados para a predição de anomalias Bouguer foram apresentados preliminarmente na Reunião SIRGAS 2010, realizada em Lima (Peru), de 11 a 12 de novembro de 2010.

\section{CONCLUSÕES E RECOMENDAÇÕES}

Nesta pesquisa inicialmente foi demonstrado que a função de Hirvonen não é capaz de modelar a covariância empírica dentro de toda a faixa de distâncias apresentada, para o caso da região de estudos. Assim, para contornar a dificuldade, foram utilizadas outras funções para ajustar a covariância empírica, chamadas de funções covariância locais. As funções que apresentaram melhor ajuste visual associado aos melhores estimadores de qualidade $\mathrm{R}^{2}$ foram às funções de Fourier de 3. ${ }^{\text {a }}$ ordem e polinomial de 4 . $^{\text {a }}$ ordem.

A função de Fourier de $3^{\mathrm{a}}$ ordem apresentou um estimador $\mathrm{R}^{2}$ de 0,97 , seguida pela função polinomial de $4^{\mathrm{a}}$ ordem com um $\mathrm{R}^{2}$ de 0,95 . A análise em termos do Erro Médio Quadrático na comparação com os valores de anomalias da gravidade Bouguer disponíveis para a checagem foram de 1,146 mGal e de 2,295 mGal, para a função polinomial e para a função de Fourier, respectivamente. Estes resultados revelaram que o $\mathrm{R}^{2}$ pode não ser um indicador de qualidade adequado para o ajuste de Funções Covariância considerando o conjunto de dados apresentado.

Também se apresentou o comportamento da função utilizada pelo programa PREDGRAV, recomendado pelo SIRGAS para predição de anomalias da gravidade contra a Covariância Empírica calculada para a região de estudos. Com relação a este particular, ainda estão sendo investigadas as idéias de como a função apresentada por Sánchez (Comunicação Pessoal) pode influenciar nos resultados finais da predição. Todavia, foi possível visualizar que a função por último citada não é uma boa modeladora da covariância empírica gerada. Portanto, recomenda-se um estudo pormenorizado do efeito das funções covariâncias para distâncias maiores, o que depende, invariavelmente, de uma base de dados com maior amplitude geográfica.

Salienta-se que uma melhoria nos resultados da predição das anomalias pode ser alcançada utilizando anomalias completas de Bouguer, subtraindo-se os efeitos topográficos ( $c f$. ., CLYDE et al., 1984). Ressalta-se ainda que pode-se remover os longos e curtos comprimentos de ondas do sinal observado do valor da gravidade empregando-se um Modelo Global do Geopotencial e a Modelagem Residual do Terreno resultando em um campo suavizado passível de interpolação. Tais resultados são importantes na discussão de prover o atual sistema de altitudes brasileiro (ortométrico-normal) com significado físico. Todos os programas utilizados nos cálculos estão disponíveis em http://www.laras.ufpr.br e os autores agradecem críticas e sugestões. 


\section{AGRADECIMENTOS}

Os autores agradecem ao CNPQ, processos 143345/2009-5 e CNPQCONICET 490245/2007-2 e à Capes.

\section{REFERÊNCIAS BIBLIOGRÁFICAS}

BOMFORD, G. Geodesy. London: Oxford, 1980. $4^{\text {th }}$ Ed.

CLYDE, C.G.; TSCHERNING, C.C.; CHIN, M.M. Gravity Empirical Covariance Values for the Continental United States. Journal of Geophysical Research. 1984. v. 89, n.B9, p. 7962-7968.

FERREIRA, V.G.Solução do Tipo Brovar para o Segundo Problema de Valor de Contorno da Geodésia com Vistas à modernização de sistemas de altitudes. 2011. Tese (Doutorado) - Universidade Federal do Paraná, Setor de Ciências da Terra, Programa de Pós-Graduação em Ciências Geodésicas. Em fase de publicação.

HEISKANEN, W; MORITZ, H. Physical Geodesy. San Francisco: W.H. Freeman and Company, 1967.

HOFMANN-WELLENHOF, B.; MORITZ, H. Physical Geodesy. Bad Vöslau: Spriner-Verlag Wien, 2005.

KIAMEHR, R. The impact of lateral density variation model in the determination of precise gravimetric geoid in mountainous areas: a case study of Iran. Geophys.J.Int. 2006. n. 167. P.521-527.

KOTSAKIS, C. Least-Squares Collocation with covariance-matching constraints. Journal of Geodesy. 2007. v.81, n.10. p.661-677.

KRARUP, T. A Contribution to the Mathematical Foundation Fo Physical Geodesy. In: BORRE, K. Mathematical Foundation of Geodesy - Selectec papers of Torben Krarup. Berlin: Springer-Verlag, 2006.

LUZ, R. T. L. Estratégias para modernização da componente vertical do Sistema Geodésico Brasileiro e sua integração ao SIRGAS. 207f. Tese (Programa de Pós-Graduação em Ciências Geodésicas). Universidade Federal do Paraná, 2008.

MORITZ, H. Advanced Least-Square Methods. Reports of the Department of Geodetic Science - Report No. 175. The Ohio State University: Columbus, 1972.

MORITZ, H. Least-Squares Collocation as a gravitational inverse problem. Reports of the Department of Geodetic Science - Report No. 249. The Ohio State University: Columbus, 1976.

PEREIRA, R.A.D.;DE FREITAS, S.R.C. Aspectos críticos no cálculo de um geóide gravimétrico e alternativas de abordagem. Curitiba: Programa de PósGraduação em Ciências Geodésicas.Seminário II, 2010.

SCHMIDT, G. Selected topics of adjustment computation. Curso ministrado no Programa de Pós-Graduação em Ciências Geodésicas da Universidade Federal do Paraná. 2007. 
SÁNCHEZ,L. Comunicação Pessoal. 2010.

SÜNKEL, H. Approximation of Covariance Functions by non-positive definite functions. Reports of the Department of Geodetic Science - Report No. 271. The Ohio State University: Columbus, 1978.

TIERRA, A.R.; DE FREITAS, S.R.C. Artificial neural network:a powerful tool for predicting gravity anomaly from sparse data. In: JEKELI, C.; BASTOS, L.; FERNANDES, J. International Association of Geodesy Symposia. 2005. v. 129 p. 208-213.

TORGE, W. Geodesy. Berlin: Walter de Gruyter, 2000.

VERMEER, M. Statistical Methods in Geodesy. Disponível em $<$ http://users.tkk.fi/mvermeer/tasj_en.pdf> . Acesso em 18/11/2010.

ZHANG, L.;LI, F.;CHEN, W.;ZHANG, C. Height datum unification between Shenzhen and Hong Kong using the solution of the linearized fixedgravimetric boundary value problem. Journal of Geodesy, 2008. v. 83, n.5, p.411-417.

(Recebido em dezembro de 2010. Aceito em junho de 2011.) 